\title{
Bouveret's Syndrome: A Rare Cause of Biliary Iléus
}

Zhar Jamal ${ }^{1 *}$, Faouzi Laytimi², El Bouhaddouti Hicham ${ }^{1}$, Ouadii Mouaqit ${ }^{1}$, Benjelloun El Bachir ${ }^{1}$, Abdelmalek Ousadden $^{1}$, Ait Taleb Khalid ${ }^{1}$

${ }^{1}$ Department of General Surgery A, CHU Hassan II FES, 30000, Morocco

${ }^{2}$ Department of General Surgery, CHU Hassan II FES, 30000, Morocco

DOI: $\underline{10.36347 / \mathrm{sasjs} .2020 . v 06 i 04.004}$

| Received: 23.03.2020 | Accepted: 01.04.2020 | Published: 08.04.2020

*Corresponding author: Zhar Jamal

\section{Abstract}

Case Report

Bouveret syndrome is is a pyloroduodenal obstruction by a gallstone migrated through a cholecystoduodenal fistula. It's a rare complication of cholelithiasis.Given the relative rarity of this condition, we review the diagnosis and management including a report of one case with an acute pancreatitis. The abdominal computed tomographic disclosed a huge gastric stasis upstream from a very tight postbulbar stenosis. The surgical approach consists on open dudenotomy and simultaneous cholecystectomy and fistula repair.

Keywords: Bouveret's syndrome; cholecystitis, gallstone ileus, surgery.

Copyright @ 2020: This is an open-access article distributed under the terms of the Creative Commons Attribution license which permits unrestricted use, distribution, and reproduction in any medium for non-commercial use (NonCommercial, or CC-BY-NC) provided the original author and source are credited.

\section{INTRODUCTION}

Bouveret syndrome is a rare form of gallstone ileus secondary to an acquired fistula between the gallbladder and either the duodenum or stomach, complicating only $0.3 \%-0.5 \%$ of patients with cholelithiasis [1]. It represents $\sim 1 \%-3 \%$ of cases of gallstone ileus. Despite the rarity of Bouveret's syndrome, it can cause notable morbidity and mortality rates [2]. Due to the rare nature of this disease, there are no standardized recommendations for the diagnostic workup and management of these patients, including endoscopic, laparoscopic, and open surgical options. We report a description of a patient (age) who was managed in our department.

\section{Case}

A 53-year-old woman with no past medical history presented to the emergency department after acute epigatric pain and vomiting. On His abdominal exam was significant for some tenderness to palpation in the epigastrium. On initial laboratory evaluation, she had a white cell count of $10 \mathrm{~K} / \mathrm{CMM}$ and a hemoglobin level of $11 \mathrm{~g} / \mathrm{d}$, lipase 400UI/L.

She was taken for esophogastroduodenoscopy (EGD) and was found a dilated stomach fistula between the gallbladder and the duodenum.
Computed tomography (CT) demonstrated findings consistent with acute pancreatiis with a large $4 \mathrm{~cm}$ gallstone in the second duodenum, sclerosus et atrophicus gallbladder (Fig-1).

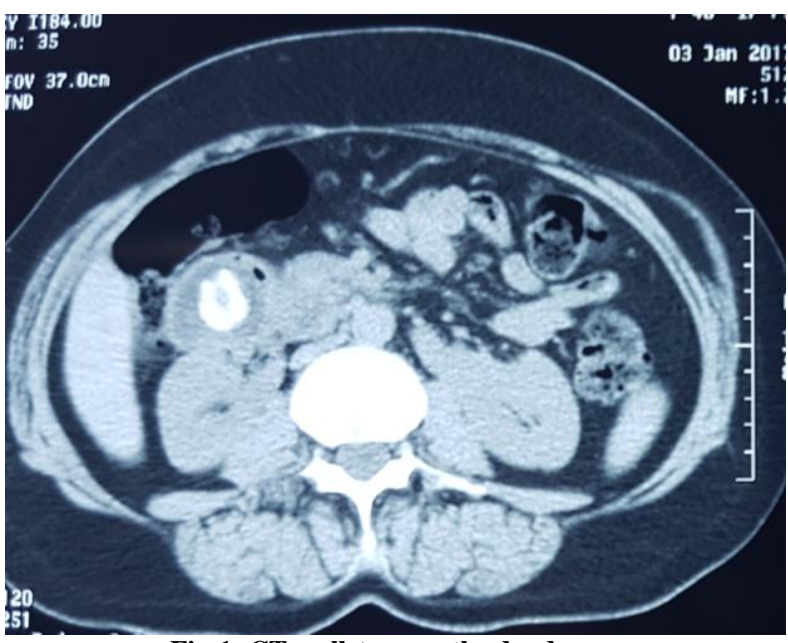

Fig-1: CT: gallstone on the duodenum

The patiente underwent open cholecystectomy with fistula repair. The gallstone was extracted through the fistula and reparated. Postoperatively, the patiente had delayed return of gastric function without incidents (Fig $2 \& 3$ ). 


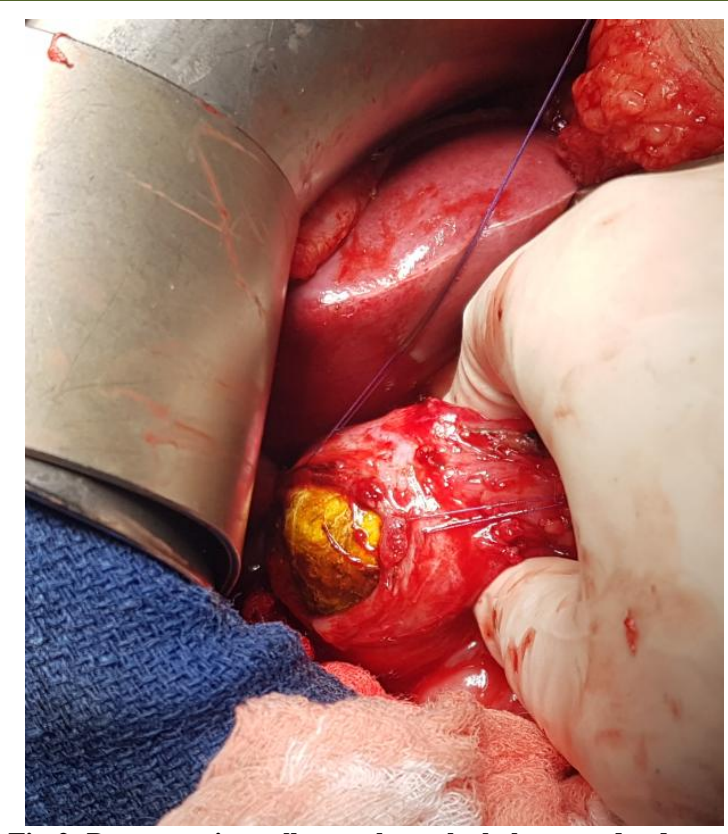

Fig-2: Per-operative gallstone through cholecysto- duodenum fistula

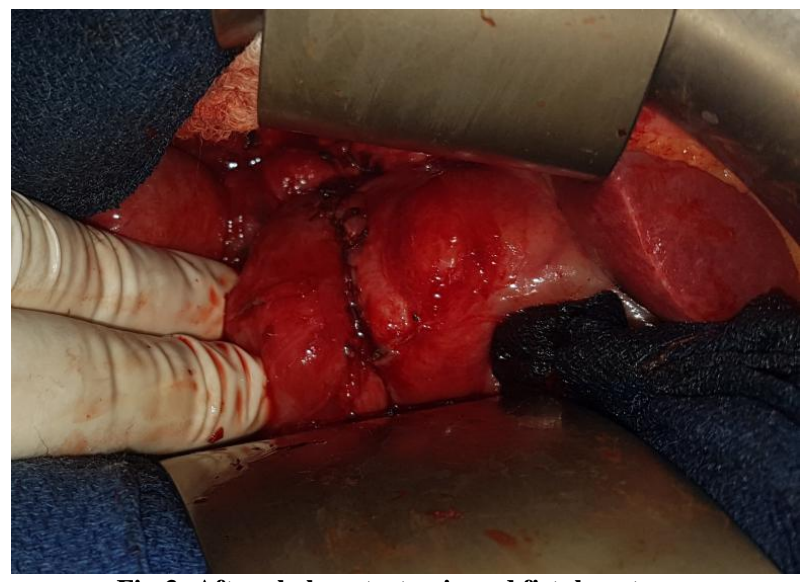

Fig-3: After cholecystectomie and fistula sutures

\section{DISCUSSION}

The first two cases of this rare condition were reported by Leon Bouveret [3]. The key to diagnosing patients with Bouveret syndrome is maintaining a high level of suspicion in patients with a history of cholelithiasis and symptoms of gastric outlet obstruction older woman a history of cholelithiasis [4].

Bouveret's syndrome usually presents with non-specific symptoms, most commonly a triad of epigastric pain, nausea, and vomiting. Differential diagnosis of gastric outlet obstruction in elderly people is subdivided into three groups: inflammatory, malignant, and congenital. Variants of this syndrome have been described in the literature, including pyloric obstruction by a gallstone passed through a cholecystogastric fistula, and external compression by a stone located outside the duodenum [5].

Most patients presenting for a suspected gastrointestinal tract obstruction are screened with an abdominal X-ray, which is diagnostic in only $21 \%$ of cases, findings suggestive of Bouveret syndrome include Rigler's triad. In most cases, MRI or computed tomography scan is needed for the diagnosis, and to view its elaborated evaluation of the fistulas, gallstones and inflammatory findings, its $93 \%$ sensitivity, $100 \%$ specificity, and $99 \%$ accuracy. Oral contrast can be added which may demonstrate extravasation of contrast into the gallbladder. More invasive diagnostic techniques include esophagogastroduodenoscopy (EGD) and surgery, both of which have diagnostic and therapeutic potentials, but Surgery serves as a diagnostic and therapeutic modality par excellence.

Treatment options for patients include a variety of endoscopic or a percutaneous approach for elderly patients with multiple medical co-morbiditiesas well as surgery. The surgery is the mainstay of treatment for gallstone ileus. The endoscopic and percutaneous methods include mechanical lithotripsy, laser lithotripsy, extracorporeal shock wave lithotripsy, and intracorporeal electrohydraulic lithotripsy [6]. The first case of successful endoscopic treatment of Bouveret syndrome was reported by Bedogni in 1985 [7].

The main surgical approaches are enterolithotomy and gastrotomy or immediately proximal to the site of obstruction with resection of irreversibly damaged parts of the small bowel. Simultaneous Cholecystectomy with fistula repair in combination or following open or laparoscopic treatment of Bouveret syndrome remains an area of debate. The advantage of this approach is prevention of subsequent biliary complications including cholecystitis and cholangitis, and gallbladder carcinoma. The best approach is the one tailored to each patient, with the consideration of his medical condition, age, comorbidities, life expectancy, and operator experience [7].

\section{CONCLUSION}

Bouveret's syndrome is a rare cause of gastric outlet obstruction by large gallstones that reach the digestive tract through a cholecystoduodenal fistula. Symptoms are non-specific and the typical radiographic triad of intestinal obstruction, ectopic gallstone, and pneumobilia is not uniformly present, and best visualized by CT scan. Therapeutic approach includes three options: a rarely successful endoscopic approach, a technically difficult mechanical and intracorporeal lithotripsy, and an almost always needed surgical approach.

\section{RÉFÉRENCES}

1. Qasaimeh GR, Bakkar S, Jadallah K. Bouveret's syndrome: an overlooked diagnosis. A case report and review of literature. International surgery. 2014 Nov;99(6):819-23. 
2. Haddad F, Mansour W, Deeb L. Bouveret's Syndrome: Literature Review. Cureus, 2018 March;10(3):e2299.

3. Bouveret L. Sténose du pylore adhérant à la vésicule. Rev Med (Paris), 1896;16:1-16.

4. Caldwell KM, Lee SJ, Leggett PL, Bajwa KS, Mehta SS, Shah SK. Bouveret syndrome: current management strategies. Clinical and experimental gastroenterology. 2018;11:69-75.
5. Park SH, Lee SW, Song TJ: Another new variant of Bouveret's syndrome. World J Gastroenterol. 2009, 15:378-379.

6. Zhao JC, Barrera E, Salabat M, Denham W, Leung D, Ujiki M. Endoscopic treatment for Bouveret syndrome. Surg Endosc. 2013;27(2):655.

7. Bedogni G, Contini S, Meinero M, Pedrazzoli C, Piccinini GC. Pyloroduodenal obstruction due to a biliary stone (Bouveret's syndrome) managed by endoscopic extraction. Gastrointest Endosc. 1985;31(1):36-38. 